\title{
Stable Bipedal Walking with Foot Rotation Through Direct Regulation of the Zero Moment Point
}

\author{
C. Chevallereau Member,IEEE, , D. Djoudi, and J.W. Grizzle Fellow,IEEE,
}

\begin{abstract}
Consider a biped evolving in the sagittal plane. The unexpected rotation of the supporting foot can be avoided by controlling the zero moment point or ZMP. The objective of this study is to propose and analyze a control strategy for simultaneously regulating the position of the ZMP and the joints of the robot. If the tracking requirements were posed in the time domain, the problem would be underactuated in the sense that the number of inputs would be less than the number of outputs. To get around this issue, the proposed controller is based on a pathfollowing control strategy previously developed for dealing with the underactuation present in planar robots without actuated ankles. In particular, the control law is defined in such a way that only the kinematic evolution of the robot's state is regulated, but not its temporal evolution. The asymptotic temporal evolution of the robot is completely defined through a one degree of freedom subsystem of the closed-loop model. Since the ZMP is controlled, bipedal walking that includes a prescribed rotation of the foot about the toe can also be considered. Simple analytical conditions are deduced which guarantee the existence of a periodic motion and the convergence towards this motion.
\end{abstract}

\section{INTRODUCTION}

The majority of robot control policies are built around the notion of controlling the Zero Moment Point (ZMP) [28], [11], [17], [20], [18], [26]. The center of pressure or CoP is a standard notion in mechanics that was renamed the ZMP by Vukobratovic and co-workers [31], [30]. As long as the ZMP remains inside the convex hull of the foot support region, $C o P=Z M P$ and the supporting foot does not rotate. In particular, most of the control strategies are decomposed into a low-level controller and a high-level controller, where the lowlevel controller ensures the tracking of the reference motion for each joint, and the high-level controller modifies the reference motion in order to ensure that the ZMP remains strictly within the convex hull of the foot support region.

In experimental studies, how to modify the reference motion is not always explained [11]. Kagami et al. [14] showed how to retouch a carelessly drawn reference into a physically consistent motion with dynamical constraints including that about ZMP taken into account. Also, Sugihara and Nakamura [25] proposed the way to re-track the originally consistent reference when a robot motion is perturbed. It guarantees that ZMP moves in the vicinity of the reference in order to stabilize the whole body motion. Obviously, the modification of the

The work of C.Chevallereau and D. Djoudi was supported by ANR grants for the PHEMA project. The work of J.W. Grizzle was supported by a National Science Foundation grant, ECS 0600869.

C. Chevallereau and D.Djoudi are with the IRCCyN, Ecole Centrale de Nantes, UMR CNRS 6597, BP 92101, 1 rue de la Noë, 44321 Nantes, cedex 03, France, Dalila.Djoudi, Christine.Chevallereaulirccyn.ec-nantes.fr

J.W. Grizzle is with the Control Systems Laboratory, EECS Department, University of Michigan, Ann Arbor, Michigan 48109-2122, USA, grizzle@umich.edu reference motion has an important effect on the stability of the gait (in the sense of the convergence toward a periodic motion) and its robustness (in the sense of the reaction of the robot in the presence of perturbations).

The existence and stability of a periodic orbit depend on much more than just the position of the ZMP: It is quite possible to have gaits where the ZMP is within the convex hull of the foot support region and where the robot remains upright, but yet the gait is not periodic, or it is periodic, but is not asymptotically stable [7].

A stability analysis of a flat-footed walking gait for a fivelink biped with an actuated ankle was carried out numerically in [12], [13], using the Poincaré return map. The unilateral constraints due to foot contact were carefully presented. Motivated by energy efficiency, a feedback controller was developed in [24] that allows a fully actuated bipedal robot walking on a flat surface to realize a passive walking gait, that is, a gait corresponding to walking down a slope without actuation. Stability of the resulting walking motion has been rigorously established, though realistic constraints on the ZMP were not imposed.

Our control strategy is based on a path-following control strategy previously developed for dealing with the underactuation present in planar robots without actuated ankles [4], [5], [10], [33]. Our controller is related to the work in [7], [6] and [32, Chap. 10], which extended the work of Westervelt et al. [33] on underactuated bipedal walking to the case of a fully actuated robot where the walking gait allowed foot rotation. In that work, the stance ankle torque was used to regulate either the position of the stance ankle or the rate of convergence to a periodic walking gait. In the present study, the position of the ZMP will instead be prescribed, which is important for robustly avoiding unexpected rotations of the foot in the presence of perturbations or for taking into account desired rotation of the supporting foot toward the end of the single support phase. This new approach has the advantage of building a link between the classical approach to the control of fully actuated bipeds based on the ZMP and the study of underactuated bipedal robots (either passive bipeds or robots with a point foot contact) based on Poincaré stability analysis. It is shown that when the ZMP position is controlled, the robot can be viewed as an underactuated mechanism and that the introduction of a foot-rotation phase in this context is straightforward.

The control law is defined in such a way that only the kinematic evolution of the robot's joints and the ZMP position are controlled, but not their temporal evolution. This strategy can be seen as an on-line modification of the joint reference motion with respect to time in order to ensure that the position of the ZMP will be satisfactory. The modification of the 
reference motion corresponds to adjusting the acceleration of the robot along a given path ${ }^{1}$ in the joint space. Assuming a perfect robot model, and without external perturbations, the closed-loop temporal evolution of the robot is completely defined and can be analyzed through the study of a one degree of freedom subsystem. The Poincaré return map can be used to study the existence and stability of periodic motions under the proposed control law. Analytical conditions are obtained and subsequently illustrated through simulations.

The modification of the reference motion can be related to the work presented in [25], since the ZMP evolution is prescribed. However two main differences appear in our work. The modification of the joint motion to obtain the desired ZMP evolution is provided by a temporal modification only, the joint path is preserved. Since our references for the joint and the ZMP position are not functions of time, the control does not attempt to re-synchronize its motion with time.

Since the control method allows a desired evolution of the ZMP to be prescribed, a desired rotation of the supporting foot about the toe during walking can be taken into account. During this sub-phase, the ZMP is placed at the forward limit of the foot and the kinematic evolution of the internal joints is prescribed, but the temporal (dynamic) evolution of the joints is determined by the controller to be compatible with the model, which, in this case, is really underactuated; the technique of [5] is used to address the underactuation. Conditions for the existence of a periodic motion and for the stability of a periodic gait that includes a prescribed rotation of the foot about the toe are also given.

The approach developed in [7], [6] and [32, Chap. 10] considers also a walking gait with foot rotation. The work in [3] further elaborates on the Poincaré stability analysis of walking gaits that include foot-rotation; in particular, the issue of the state dimension varying from one phase to another is emphasized. For walking gaits that include foot rotation, various ad hoc control solutions have been proposed in the literature [15], [16], [22], [27], [34], but none of them can guarantee stability in the presence of the underactuation that occurs during toe roll.

The first part of the paper considers only flat-footed walking. Section II presents the dynamic model of the biped. A planar biped is considered. Section III is devoted to the formulation of the control strategy. In Section IV, a complete analytical study of stability is proposed. Some simulation results are presented in Section V; a response to a perturbation with a known model of the robot is considered as well as a response with an uncertain model of the biped. Section VI proposes an extension of the control law considering a gait that includes a prescribed rotation of the supporting foot; a simulation illustrates the theoretical results. Section VII concludes the paper.

\section{THE BIPED MOdEL}

\section{A. The biped}

The biped under study walks in the sagittal plane identified with a vertical $x-z$-plane. The robot is comprised of a

\footnotetext{
${ }^{1}$ The time evolution along the path is not specified a priori. For related work in nonlinear control, see [1] and references therein.
}

\begin{tabular}{|c|r|r|r|r|}
\hline & feet & femur & tibia & torso \\
\hline \hline length in $\mathrm{m}$ & 0.26 & 0.4 & 0.4 & 0.625 \\
\hline mass in $\mathrm{kg}$ & 1 & 3.2 & 6.8 & 17 \\
\hline inertia in $\mathrm{kg} \mathrm{m} \mathrm{m}^{2}$ & 0.012 & 0.048 & 0.069 & 1.869 \\
\hline
\end{tabular}

TABLE I

BIPED PARAMETERS FOR SIMULATION.

torso and two identical legs, and each leg is composed of two links and a foot. The ankles, the knees and the hips are onedegree-of-freedom rotational frictionless joints. The walking gait consists of single support phases where the stance foot is flat on the ground separated by impacts, that is, instantaneous double support phases where leg exchange takes place. The vector $q=\left[q_{1}, q_{2}, q_{3}, q_{4}, q_{5}, q_{6}\right]^{T}$ of configuration variables (see Figure 1) describes the shape and orientation of the biped during single support. The torques are grouped into a torque vector, $\Gamma=\left[\Gamma_{1}, \Gamma_{2}, \Gamma_{3}, \Gamma_{4}, \Gamma_{5}, \Gamma_{6}\right]^{T}$.

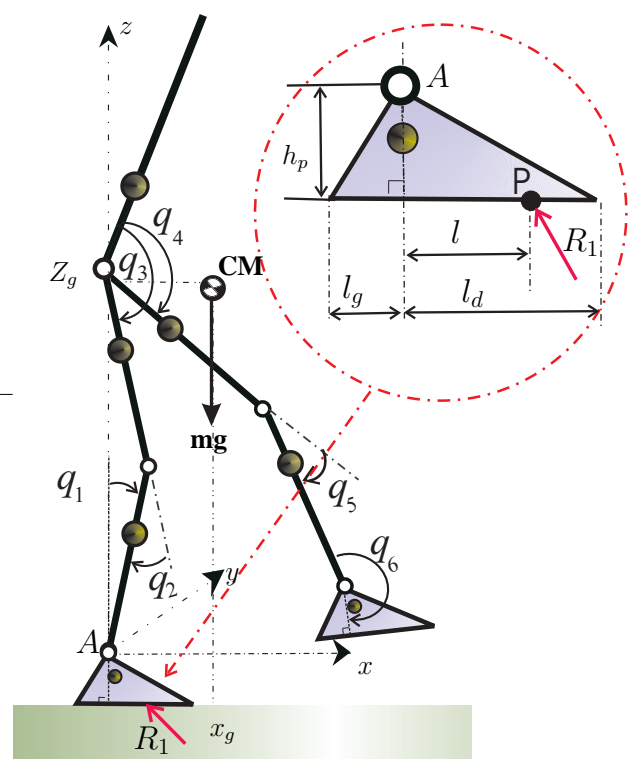

Fig. 1. The studied biped and a choice of generalized coordinates.

In the simulation, we use the biped parameters given in $\mathrm{T}$ able I. The dimensions of the feet are $h_{p}=0.08 \mathrm{~m}, l_{g}=$ $0.06 \mathrm{~m}$ and $l_{d}=0.2 \mathrm{~m}$.

\section{B. Dynamic model}

The walking gait is composed of successive phases of single support and instantaneous double support. A passive impact exists at the end of the single support phase. The legs swap their roles from one step to the next, and thus, because the robot is symmetric, the study of a single step is sufficient to deduce the complete behavior of the robot over a sequence of steps on alternating legs. Only the dynamic model for support on leg-1 is presented here.

1) The single support phase model: The dynamic model can be written as follows:

$$
M(q) \ddot{q}+h(q, \dot{q})=\Gamma,
$$


where $M(q)$ is a $(6 \times 6)$ matrix and the vector $h(q, \dot{q})$ contains the centrifugal, Coriolis and gravity forces.

2) Global equilibrium in translation: The reaction force during the single support phase: During single support, the position of the center of mass of the biped can be expressed as a function of the angular coordinates and is denoted by $x_{g}(q)$, $z_{g}(q)$. When leg- 1 is on the ground, a ground reaction force $R_{1}$ exists. The global equilibrium in translation of the robot makes it possible to calculate this force. Thus we have

$$
m\left[\begin{array}{c}
\ddot{x}_{g} \\
\ddot{z}_{g}
\end{array}\right]+m g\left[\begin{array}{l}
0 \\
1
\end{array}\right]=R_{1}
$$

Equation (2) can also be written as

$$
\begin{aligned}
m \frac{\partial x_{g}(q)}{\partial q} \ddot{q}+m \dot{q}^{T} \frac{\partial^{2} x_{g}(q)}{\partial q^{2}} \dot{q} & =R_{x 1} \\
m \frac{\partial z_{g}(q)}{\partial q} \ddot{q}+m \dot{q}^{T} \frac{\partial^{2} z_{g}(q)}{\partial q^{2}} \dot{q}+m g & =R_{z 1},
\end{aligned}
$$

where $\frac{\partial^{2} x_{g}(q)}{\partial q^{2}}$ and $\frac{\partial^{2} z_{g}(q)}{\partial q^{2}}$ are $(6 \times 6)$ matrices.

3) Global equilibrium in rotation: the ZMP position: The robot is submitted to the reaction force exerted by the ground at the ZMP, and the force of gravity. Since the stance ankle $A$ is stationary during the single support phase, the equilibrium of the foot around the axis of the ankle can be written (see Figure 1):

$$
\dot{\sigma}_{A}=m g x_{g}-l R_{z 1}-h_{p} R_{x 1},
$$

where $\sigma_{A}$ is the angular momentum of the biped about $A$. By definition, the angular momentum is linear with respect to the joint velocities and can be written

$$
\sigma_{A}=N(q) \dot{q}
$$

The location of the ZMP is then defined directly by the robot dynamics through the previous equation. Indeed, using (3), (4) and (5), we have

$$
\left(N_{0}(q)+l N_{l}(q)\right) \ddot{q}+h_{0}(q, \dot{q})+l h_{l}(q, \dot{q})=0,
$$

where

$$
\begin{aligned}
N_{0} & =N(q)+m h_{p} \frac{\partial x_{g}(q)}{\partial q} \\
N_{l} & =m \frac{\partial z_{g}(q)}{\partial q} \\
h_{0} & =\dot{q}^{T} \frac{\partial N(q)}{\partial q} \dot{q}-m g x_{g}(q)+m h_{p} \dot{q}^{T} \frac{\partial^{2} x_{g}(q)}{\partial q^{2}} \dot{q} \\
h_{l} & =m \dot{q}^{T} \frac{\partial^{2} z_{g}(q)}{\partial q^{2}} \dot{q}+m g .
\end{aligned}
$$

Equation (4) can also be rewritten, using (2), in the form

$$
\frac{d\left(\sigma_{A}+l\left(m \dot{z}_{g}\right)+h_{p}\left(m \dot{x}_{g}\right)\right)}{d t}-m i \dot{z}_{g}-m g\left(x_{g}-l\right)=0 .
$$

By definition, the term $\sigma_{A}+l\left(m \dot{z}_{g}\right)+h_{p}\left(m \dot{x}_{g}\right)$ is the angular momentum about the ZMP with $x$-coordinate $l$, which is denoted here by $\sigma_{P}$. This equation, corresponding to angular momentum balance, can be also written as ${ }^{2}$

$$
\dot{\sigma}_{P}=m \dot{l} \dot{z}_{g}+m g\left(x_{g}-l\right)
$$

\footnotetext{
${ }^{2}$ The equilibrium in rotation of the robot gives directly this equation, the term $m \dot{l} \dot{z}_{g}$ comes from the fact that the ZMP is not stationary.
}

\section{The impact model}

When the swing leg (i.e., leg-2) touches the ground with a flat foot at the end of the single support phase, an impact takes place. The velocity of foot- 2 becomes zero just after the impact. We study a gait with instantaneous double support so that, just after the impact, the former stance leg-1 lifts off the ground. The robot's configuration $q$ is assumed to be constant during the instant of double support, while there are jumps in the velocities. The velocity vectors just before and just after impact, are denoted $\dot{q}^{-}$and $\dot{q}^{+}$, respectively, where ${ }^{+}$means after the impact and ${ }^{-}$before the impact. The impact model can be written as [5]

$$
\dot{q}^{+}=E\left(\triangle(q) \dot{q}^{-}\right),
$$

where $\triangle(q)$ is a $6 \times 6$ matrix, and $E$ is the permutation matrix describing leg exchange. For the ensuing single support phase, the joints are relabelled in order to limit the analysis to a single dynamic model for single support.

\section{The Control Strategy}

The desired walking gait is assumed to be composed only of single support phases where the stance foot is flat on the ground and stationary (i.e., it neither rotates nor slips). While a flat-footed gait is not a necessary condition for walking as we will see in Section VI, we focus our attention in this part on fully actuated phases. Direct control of the position of the ZMP will prevent unwanted foot rotation, and thus a desired ZMP position, $l^{d}$, is prescribed [9]. As shown in the previous section, the position of the ZMP is directly connected to the acceleration of the robot's motion. It is therefore impossible to prescribe independently a desired evolution of the joints, $q^{d}(t)$, and the position of the ZMP, $l^{d}(t)$. With respect to such a task, the biped can be seen as an underactuated system. Thus, as in [5], the objective of the control law presented in this section is not to track a (time-based) reference motion for $q$ and $l$, but only the associated path in joint space. A reference motion differs from a path by the fact that a motion is a temporal evolution along a path. A joint path is the projection of a joint motion in the joint space. The difference between a motion and a path is illustrated in Figure 2 for a two-link robot.

Only tracking of the desired path is sought and a timescaling control law as in [8] is used. Reference paths for the joints and ZMP, $q^{d}(s)$ and $l^{d}(s)$, respectively, are assumed to be known as a function of a scalar path parameter $s$, which plays the role of a normalized virtual time. A desired gait of the robot corresponds to specification of $s$ as an increasing function of time, $s(t)$.

\section{A. Requirements for a Feasible Reference Path}

The reference path $q^{d}(s), l^{d}(s)$ is designed in order to be compatible with a periodic solution of the biped model. The legs swap their roles from one step to the next, so the reference path can be defined for one step only. For the first step, the scalar path parameter $s$ increases strictly monotonically with respect to time from 0 to 1 and impact takes place at $s=1$. The evolution of $s$ for step $k$ is denoted $s_{k}(t)$. 


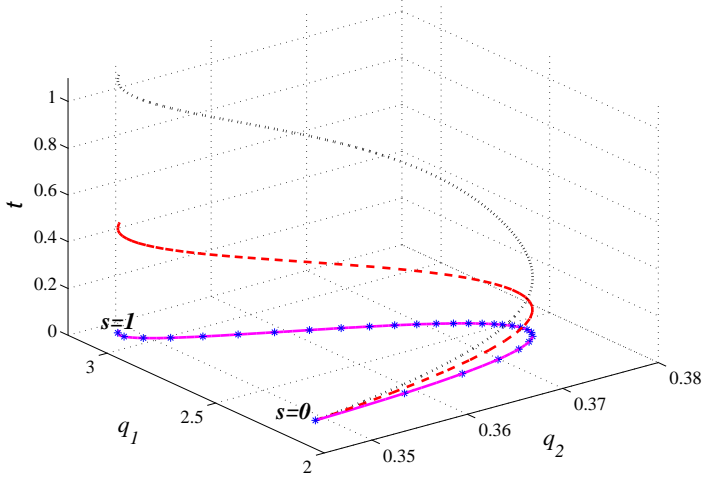

Fig. 2. The dotted lines are two motions $\left(q_{1}(t), q_{2}(t)\right)$ corresponding to the same path represented by the solid line. A path is a line in the joint space, this line can be parametrized as a function of a new variable denoted $s$, and then can be expressed by $\left(q_{1}(s), q_{2}(s)\right)$. This function $s$ is defined such that the initial configuration corresponds to $s=0$ and the final configuration corresponds to $s=1$. Any monotonic function $s(t)$ defines a motion corresponding to the path $q(s)$. For example $s=t / T$ defines a motion of duration $T$. If the desired path is such that $q_{1}$ for example is monotonic, the path can also be written as $q_{2}\left(q_{1}\right)$, and the control law proposed here can be computed in this context [32, Chap. 11].

The single support phase corresponds to $0<s<1$. Due to the leg exchange at impact, the vectors $q^{d}(0)$ and $q^{d}(1)$, describing respectively the initial and final desired positions of the biped, must be such that $q^{d}(1)=E\left(q^{d}(0)\right)$.

The initial and final velocity of the biped are connected by the impact model and leg exchange (9). The reference path is designed so that if the reference path is exactly tracked before the impact (but the robot state is not necessarily on the periodic motion), then the reference path will be exactly tracked after the impact. Just before the $k+1$-st impact, on the reference path, the vector of joint velocities is $\dot{q}^{-}=\frac{d q^{d}(1)}{d s} \dot{s}_{k}(1)$. The reference path is designed such that, after the impact, the reference path is also perfectly tracked $\dot{q}^{+}=\frac{d q^{d}(0)}{d s} \dot{s}_{k+1}(0)$. Since the impact model (9) connects the velocities before and after impact, we must have

$$
\frac{d q^{d}(0)}{d s} \dot{s}_{k+1}(0)=E \triangle\left(q^{d}(1)\right) \frac{d q^{d}(1)}{d s} \dot{s}_{k}(1) .
$$

When $q^{d}(1)$ and $\frac{d q^{d}(1)}{d s}$ are known, there are an infinite number of possible choices for $\frac{d q^{d}(0)}{d s}$. The set of solutions can be parametrized by a scalar $\alpha$ as

$$
\frac{d q^{d}(0)}{d s}=E \triangle\left(q^{d}(1)\right) \frac{d q^{d}(1)}{d s} \alpha,
$$

yielding $\dot{s}_{k+1}(0)=\frac{\dot{s}_{k}(1)}{\alpha}$.

\section{B. Definition of the control law}

The control law is selected to ensure that the joint coordinates follow the joint reference path, $q^{d}(s)$, and that the position of the ZMP is $l^{d}(s)$. The torque acts on the second derivative of $q$ and directly on $l$. It follows from the definition of the joint reference path that the desired velocity and acceleration of the joint variables are

$$
\begin{aligned}
& \dot{q}^{d}(t)=\frac{d q^{d}(s(t))}{d s} \dot{s} \\
& \ddot{q}^{d}(t)=\frac{d q^{d}(s(t))}{d s} \ddot{s}+\frac{d^{2} q^{d}(s(t))}{d s^{2}} \dot{s}^{2} .
\end{aligned}
$$

The function $s(t)$ needs to be a strictly increasing function of $t$, but because the control objective is only to track a reference path, the evolution of $s(t)$ is otherwise free and the second derivative $\ddot{s}$ can be treated as a "supplementary control input". This allows the control law to be designed for a system with equal number of inputs and outputs: The control inputs are the six torques $\Gamma_{j}, j=1, \ldots, 6$, plus $\ddot{s}$, and the chosen outputs are the six components of $q(t)-q^{d}(s(t))$ and $l(t)-l^{d}(s(t))$.

The control law is based on computed torque, which is quite commonly used in robotics, with a small modification to ensure finite-time convergence to the desired paths. The finite-time feedback function proposed in [2], [10] is used. The joint tracking errors are defined with respect to trajectories satisfying (12)

$$
\begin{aligned}
& e_{q}(t)=q^{d}(s(t))-q(t) \\
& \dot{e}_{q}(t)=\frac{d q^{d}(s(t))}{d s} \dot{s}-\dot{q}(t) .
\end{aligned}
$$

The desired behavior of the configuration variables in closed loop is

$$
\ddot{q}=\ddot{q}^{d}+\psi(q, \dot{q}, s, \dot{s}),
$$

where $\psi(q, \dot{q}, s, \dot{s})$ from [2], [10] is the term that imposes $\left(q(t)-q^{d}(s(t))\right) \rightarrow 0$ in finite time; in fact, the settling time can be chosen to be less than the time duration of a step. $\psi$ is a vector of five components $\psi_{k}, k=1, \ldots, 5$ with

$$
\psi_{k}=-\operatorname{sign}\left(\epsilon \dot{e}_{q_{l}}\right)\left|\epsilon \dot{e}_{q_{l}}\right|^{\nu}-\operatorname{sign}\left(\phi_{l}\right)\left|\phi_{l}\right|^{\nu},
$$

where $\phi_{l}=e_{q_{l}}+\frac{1}{2-\nu} \operatorname{sign}\left(\epsilon \dot{e}_{q_{l}}\right)\left|\epsilon \dot{e}_{q_{l}}\right|^{2-\nu}$, and the parameters $0<\nu<1$ and $\epsilon>0$ are used to adjust the settling time of the controller. Taking into account the expression for the reference motion, (14) can be rewritten as

$$
\ddot{q}=\frac{d q^{d}(s)}{d s} \ddot{s}+v(s, \dot{s}, q, \dot{q}),
$$

with $v(s, \dot{s}, q, \dot{q})=\frac{d^{2} q^{d}(s)}{d s^{2}} \dot{s}^{2}+\psi$. For the position of the $\mathrm{ZMP}$, the desired closed-loop behavior is

$$
l(t)=l^{d}(s(t)) .
$$

Combining expression (16) with the dynamic model (1) of the robot and the relation (6) for the ZMP determines the feedback controller. Thus, the control law must be such that

$$
\begin{aligned}
M(q)\left(\frac{d q^{d}(s)}{d s} \ddot{s}+v\right)+h(q, \dot{q}) & =\Gamma \\
\left(N_{0}(q)+l^{d}(s) N_{l}(q)\right)\left(\frac{d q^{d}(s)}{d s} \ddot{s}+v\right) & \\
+h_{0}(q, \dot{q})+l^{d}(s) h_{l}(q, \dot{q}) & =0 .
\end{aligned}
$$

It follows that, in order to obtain the desired closed-loop behavior, it is necessary and sufficient to choose

$$
\begin{aligned}
\ddot{s} & =\frac{-\left(N_{0}(q)+l^{d}(s) N_{l}(q)\right) v-h_{0}(q, \dot{q})-l^{d}(s) h_{l}(q, \dot{q})}{\left(N_{0}(q)+l^{d}(s) N_{l}(q)\right) \frac{d q^{d}(s)}{d s}} \\
\Gamma & =M(q)\left(\frac{d q^{d}(s)}{d s} \ddot{s}+v\right)+h(q, \dot{q}) .
\end{aligned}
$$

As long as $\left(N_{0}(q)+l^{d}(s) N_{l}(q)\right) \frac{d q^{d}(s)}{d s} \neq 0$, the control law (18) is well defined, and, by (14), ensures that, $q(t)$ converges to $q^{d}(s(t))$ in finite time, and that $l(t)=l^{d}(s(t))$. Without initial errors, a perfect tracking of $q^{d}(s(t))$ and $l^{d}(s)$ is obtained.

At this point, the behavior of $s(t)$ is unknown. Properties of its temporal evolution are developed next. 


\section{STABility Study}

Since the control law is designed to converge before the end of the first step, after that, perfect tracking is obtained and therefore

$$
\begin{aligned}
& q(t)=q^{d}(s(t)) \\
& \dot{q}(t)=\frac{d q^{d}(s)}{d s} \dot{s}(t) \\
& \ddot{q}(t)=\frac{d q^{d}(s)}{d s} \ddot{s}(t)+\frac{d^{2} q^{d}(s)}{d s^{2}} \dot{s}(t)^{2} \\
& l(t)=l^{d}(s(t)) .
\end{aligned}
$$

These equations define the zero dynamics manifold corresponding to the proposed control law. On the zero dynamics manifold, the evolution of $\dot{s}$ during one step can be determined by integration of the dynamic equation corresponding to the global equilibrium in rotation (8).

Since the occurrence of an impact depends only on the configuration of the robot and not its velocity, and due to the characteristics of the joint reference path (Section III-A), each step begins with $s=0$ and finishes with $s=1$.

The stability of the control law is defined in the sense of the convergence towards a periodic motion. A periodic motion of the biped corresponds to a periodic evolution of the angular momentum. Thus we study the evolution of the angular momentum from one step to the next one and deduce a condition allowing the existence of an attractive periodic solution.

\section{A. Evolution of the angular momentum for one step}

On the zero dynamics, (8) becomes

$$
\dot{\sigma}_{P}=m \frac{d l^{d}(s)}{d s} \frac{\partial z_{g}\left(q^{d}(s)\right)}{\partial q} \frac{d q^{d}(s)}{d s} \dot{s}^{2}+m g\left(x_{g}\left(q^{d}(s)\right)-l^{d}(s)\right)
$$

and

$$
\sigma_{P}(s, \dot{s})=I(s) \dot{s},
$$

with

$$
I(s)=\left(N\left(q^{d}(s)\right)+m l^{d}(s) \frac{\partial z_{g}\left(q^{d}(s)\right)}{\partial q}+m h_{p} \frac{\partial x_{g}\left(q^{d}(s)\right)}{\partial q}\right) \frac{d q^{d}(s)}{d s} .
$$

Equations $(20,21)$ can be combined to express the derivative of the angular momentum with respect to $s$.

Just as in [33, Prop. 1], it can be shown that uniqueness of solutions of $(20,21)$ implies that if the robot completes a step, that is, if there exists a solution beginning with $s(0)=0$, $\dot{s}(0)>0$ and ending with $s\left(t_{f}\right)=1, \dot{s}\left(t_{f}\right)>0$, then for $t \in\left[0, t_{f}\right], \dot{s}(t)>0$. Using this fact, $(20,21)$ can be combined to obtain

$$
\begin{aligned}
\frac{d \sigma_{P}}{d s}= & m \frac{d l^{d}(s)}{d s} \frac{\partial z_{g}\left(q^{d}(s)\right)}{\partial q} \frac{d q^{d}(s)}{d s} \frac{\sigma_{P}}{I(s)} \\
& +m g\left(x_{g}\left(q^{d}(s)-l^{d}(s)\right) \frac{I(s)}{\sigma_{P}} .\right.
\end{aligned}
$$

Applying the change of variable, $\zeta(s)=\frac{1}{2} \sigma_{P}^{2}$, this equation becomes

$$
\frac{d \zeta}{d s}=2 \kappa(s) \zeta+m g I(s)\left(x_{g}\left(q^{d}(s)-l^{d}(s)\right),\right.
$$

with $\kappa(s)=\frac{m}{I(s)^{2}} \frac{d l^{d}(s)}{d s} \frac{\partial z_{g}\left(q^{d}(s)\right)}{\partial q} \frac{d q^{d}(s)}{d s}$. The above equation is a linear s-varying ODE and has the explicit solution

$$
\zeta(s)=\delta^{2}(s) \zeta(0)-\Phi(s)
$$

where

$$
\begin{aligned}
& \delta(s)=\exp \left(\int_{0}^{s} \kappa(\tau) d \tau\right) \\
& \Phi(s)=-m g \int_{0}^{s} \exp \left(2 \int_{\tau}^{s} \kappa\left(\tau_{1}\right) d \tau_{1}\right) I(\tau)\left(x_{g}\left(q^{d}(\tau)\right)-l^{d}(\tau)\right) d \tau .
\end{aligned}
$$

\section{B. Minimal angular momentum to achieve a step}

The functions $\delta(s)$ and $\Phi(s)$ are calculated directly from $q^{d}(s)$ and $l^{d}(s)$. A complete step can be accomplished only if $\dot{s}$ is always positive. When the robot follows the reference trajectory (19), the control law (18) does not cross a singularity as long as

$$
\left(N_{0}\left(q^{d}(s)\right)+l^{d}(s) N_{l}\left(q^{d}(s)\right)\right) \frac{d q^{d}(s)}{d s}=I(s) \neq 0 .
$$

Under this assumption, the condition $\dot{s} \neq 0$ is equivalent to $\sigma_{P} \neq 0$ or $\zeta \neq 0$.

Theorem 1 Assuming (26), a step can be achieved if, and only if, the initial value of $\zeta$ for this step is such that

$$
\zeta(0)>Z_{m}=\max _{0 \leq s \leq 1}\left(\frac{\Phi(s)}{\delta^{2}(s)}\right) .
$$

C. Evolution of angular momentum during the impact phase

At the impact, due to (11), the evolution of $\dot{s}$ is such that $\dot{s}^{+}=\frac{\dot{s}^{-}}{\alpha}$. Therefore,

$$
\sigma_{P}^{+}=\delta_{I} \sigma_{P}^{-},
$$

with $\delta_{I}=\frac{I(0)}{I(1) \alpha}$, where $I$ is given in (22).

\section{Conditions for existence and uniqueness of a periodic solution}

The combination of (25) and (28) defines the evolution of $\zeta$ (or, equivalently, $\sigma_{P}$ ) from one step to the next. The evolution of the robot during one step is completely defined by the value of $\zeta$ for a single value of $s$. Thus, we study the evolution of $\zeta$ just before the impact $\zeta^{-}=\zeta(1)$ from one step to the next, via the Poincaré map

$$
\rho\left(\zeta^{-}\right)=\left(\delta_{I} \delta(1)\right)^{2} \zeta^{-}-\Phi(1) .
$$

A periodic admissible reference motion is defined by a periodic evolution of the angular momentum, which is equivalent to a fixed point of the Poincaré map $\rho$, namely, $\zeta^{*}=\rho\left(\zeta^{*}\right)$.

From (29), taking into account that $\zeta(s)>0$, it follows that - if $\left(\delta_{I} \delta(1)\right)^{2}=1$ and $\Phi(1)=0$, then any initial value $\zeta$ produces a periodic reference motion; moreover,

- the Poincaré map has a unique fixed point

$$
\zeta^{*}=\frac{-\Phi(1)}{1-\left(\delta_{I} \delta(1)\right)^{2}},
$$

if, and only if, $\Phi(1)$ and $1-\left(\delta_{I} \delta(1)\right)^{2}$ have opposite signs.

Applying Theorem 1 and using (29), $\zeta^{*}$ in (30) defines a periodic reference motion if, and only if, the periodic angular momentum is sufficient to produce the step, that is, $\left(\delta_{1}\right)^{2} \zeta^{*}>$ $Z_{m}$.

Theorem 2 Assuming (26), a unique periodic reference motion exists if, and only if $\frac{-\Phi(1)}{1-\left(\delta_{I} \delta(1)\right)^{2}}>\frac{Z_{m}}{\left(\delta_{I}\right)^{2}}$. The periodic motion is defined by equation (30). 


\section{E. Convergence Towards the Periodic Reference Motion}

Equation (29) is equivalent to

$$
\rho\left(\zeta^{-}\right)-\zeta^{*}=\left(\delta_{I} \delta(1)\right)^{2}\left(\zeta^{-}-\zeta^{*}\right) .
$$

Consequently, solutions of equations (20) and (21) converge to the periodic motion if, and only if, $\left(\delta_{I} \delta(1)\right)^{2}<1$.

Theorem 3 Assuming (26), solutions of the zero dynamics given by equations (20) and (21) converge to the periodic reference motion if, and only if, $\left(\delta_{I} \delta(1)\right)^{2}<1$.

Combining Theorems 1, 2 and 3, the following corollary is deduced.

Corollary: Assuming (26), the reference periodic motion is orbitally exponentially stable if, and only if, the reference joint path is such that $-\Phi(1)>\max \left(\frac{1-\left(\delta_{I} \delta(1)\right)^{2}}{\delta_{I}^{2}} Z_{m}, 0\right)$ and $\left(\delta_{I} \delta(1)\right)^{2}<1$

\section{Simulation Results}

\section{A. Response to a perturbation with a perfect model}

The control law is evaluated here for the periodic path depicted in the stick diagram of Figure 3. The joint path $q^{d}(s)$ is defined with a degree four polynomial in $s$. The evolution of the ZMP position is chosen to be a linear function of $s$. The reference path $l^{d}(s), q^{d}(s)$ can be the result of an optimization process since the periodic motion, if it exists, can be explicitly deduced via (30). A methodology allowing to do this is given in [32].

In the simulation presented here, the desired evolution for $l_{d}$ and $q_{d}(s)$ are :

$$
\left\{\begin{array}{l}
q^{d}(s)=a_{0}+a_{1} s+a_{2} s^{2}+a_{3} s^{3}+a_{4} s^{4} \\
l^{d}(s)=-0.02+0.1 s
\end{array}\right.
$$

The vectors $a_{i}$ are determined so as to join an initial configuration $q^{d}(0)$, an intermediate configuration $q^{d}(0.5)$, a final configuration $q^{d}(1)$, an initial velocity $\frac{q^{d}(0)}{d s}$ and a final velocity $\frac{q^{d}(1)}{d s}$. The final configuration for the step $q_{r}^{d}(1)$ corresponds to the initial one $q^{d}(0)$ with an exchange of the leg. These key values which are used to describe the reference path are given in Table II.

\begin{tabular}{|c|c|c|c|c|}
\hline$q^{d}(0)$ & $\frac{q^{d}(0)}{d s}$ & $q^{d}(0.5)$ & $q^{d}(1)$ & $\frac{q^{d}(1)}{d s}$ \\
\hline 0.0360 & 0.9570 & 0.2014 & 0.4057 & 0.6309 \\
0.5432 & 0.2160 & 0.2239 & 0.1000 & 0.2928 \\
2.3705 & 0.6371 & 2.8350 & 3.1855 & 0.4259 \\
3.1855 & 0.3981 & 2.7072 & 2.3705 & 0.0063 \\
0.1000 & 0.5476 & 0.6938 & 0.5452 & -1.1129 \\
2.7359 & -1.0496 & 2.5981 & 3.1056 & 1.1944 \\
\hline
\end{tabular}

TABLE II

THE REFERENCE FOR THE JOINT ARE BASED ON THESE KEY VECTORS.

The evolution of $\delta(s)$ and $\Phi(s)$ are given in Figure 4. Their final values, $\Phi(1)=-205$, and $\delta(1)^{2}=0.9954$ are useful for constructing the Poincaré map. The behavior of $\zeta$ during the impact is defined by $\delta_{I}^{2}$, which is equal to 0.6422 . The minimal value of $\zeta$ for which a step can be achieved is $\frac{Z_{m}}{\delta_{I}}=$

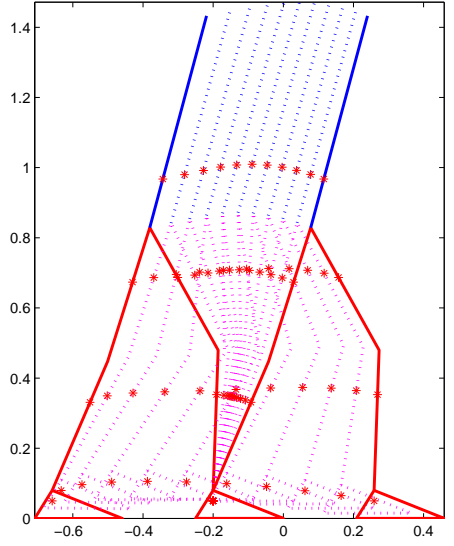

Fig. 3. The stick diagram of the nominal periodic trajectory. The configuration of the robot is drawn for $s=0,0.1,0.2 \ldots, 0.9,1$. Thus a sequence of snapshots of the robot is given. The desired motions of the robot are such that the configuration of the robot coincides at some instant to each snapshot, but it is not imposed that these instants are equally distributed within the period of one step.
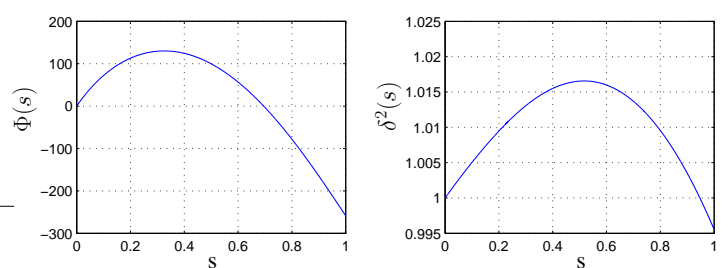

Fig. 4. The evolution of $\zeta$ during the single support phase is characterized by the functions $\Phi(s)$ and $\delta^{2}(s)$. The position of the ZMP is being controlled to increase linearly, from back to front. Because the vertical component of the velocity of the center of mass is directed upward at the beginning of the step and then downward, $\delta(s)$ increases at the beginning of the step and then decreases. Because the center of mass is behind the ZMP at the beginning of the step and then in front of the ZMP, $\Phi(s)$ increases at the beginning of the step and then decreases.

232. The periodic motion is given by (30). The fixed point occurs at $\zeta^{*}=569$. The slope of the Poincaré return map $\rho$ is $\left(\delta(1) \delta_{I}\right)^{2}=0.6393$, and because it is less than 1 , the corresponding periodic walking motion is exponentially stable. The stability arises from the effect of the impact because $\delta(1)^{2}$ is close to 1 .

A simulation was done for ten steps, assuming no modeling error. The state of the robot was initialized on the periodic orbit and horizontal force $(350 \mathrm{~N})$ was applied for $0.2 s<t<0.24 s$ at the center of mass; see Figure 5. Convergence toward a periodic motion was obtained for each of the five joints of the robot. As an illustration, the evolution of the angle of the torso is depicted in Figure 5-a. The same convergence is also evident in the evolution of the position of the ZMP with respect to time in Figure 5-b; for each step, its evolution is linear from $-0.02 m$ to $0.08 m$ except when the perturbation exists. Figure 5-c presents the evolution of $\dot{s}$ with respect to time; it clearly converges toward a periodic motion.

\section{B. Response to a perturbation with imprecise model data}

In practice, the robot's parameters are not perfectly known. We assume that we have some errors on the masses and on 

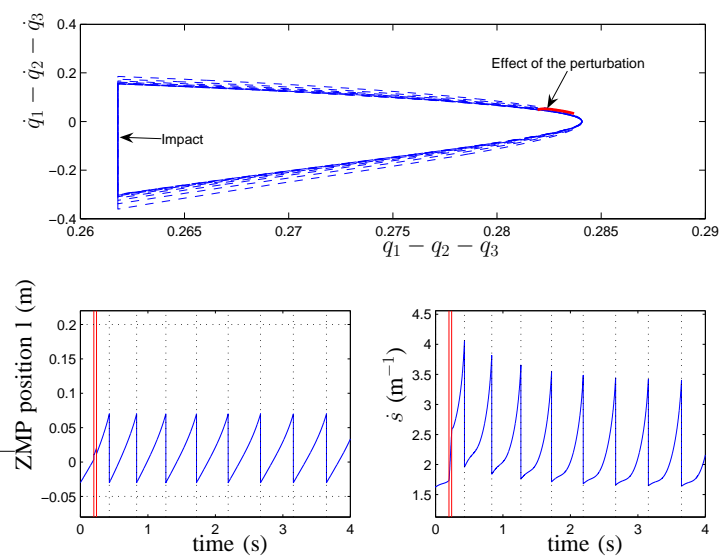

Fig. 5. The convergence towards a periodic motion is observed in simulation with the proposed control law.

the inertias of the links. We simulate the following cases of error:

- The mass errors are $+10 \%$ for the feet, $+30 \%$ for the thighs, $+30 \%$ for the shanks and $+40 \%$ for the torso. The error on the inertia of the torso is $+30 \%$. This choice of errors is arbitrary. We have chosen that the real robot is heavier than the model used in the control law; this point is commented upon in the sequel.

- Since the reference path is designed with an incorrect model, the velocity after the impact does not correspond to the expected value.

- Because the position $l$ of the ZMP is calculated via the dynamic model, $l(s)$ will not be exactly equal to $l^{d}(s)$.

Initializing the state of the robot on the theoretical periodic motion, the behavior obtained for a large number of steps is presented in Figure 6. Some tracking errors exist, particularly at the beginning of each step, due to the effect of the impact, thus the path followed is not exactly the expected one (but the tracking errors are periodic). The convergence toward a periodic motion is shown for the torso evolution via its phase plane in Figure 6-a. This convergence is also illustrated via the evolution of $\dot{s}$ with respect to s in Figure 6-c, which clearly converges toward a stable periodic motion. The periodic motion is close to the expected one but not exactly the same, because it is the result of the motion of the ZMP and of the real dynamic model. Since the real robot is heavier than the employed model of the robot, we have greater ground reaction forces; consequently the real evolution $l$ of the ZMP in Figure 6-b varies between extreme values that are smaller in absolute value than the desired values. The difference between $l(s)$ and $l^{d}(s)$ is higher for larger values of $\dot{s}$. In the case examined here, there is no problem because constraints on the equilibrium of the supporting foot are always satisfied. On the other hand, if the real robot were lighter than the modeled one, the ZMP could be at one of the extreme ends of the foot, thereby violating the constraints of equilibrium of the supporting foot. Hence, a safety margin is necessary when the minimum and the maximum values for the ZMP evolution are defined. The best way is to define $l_{\min }$ and $l_{\max }$ with some margins with respect to the actual size of the foot (see Figure
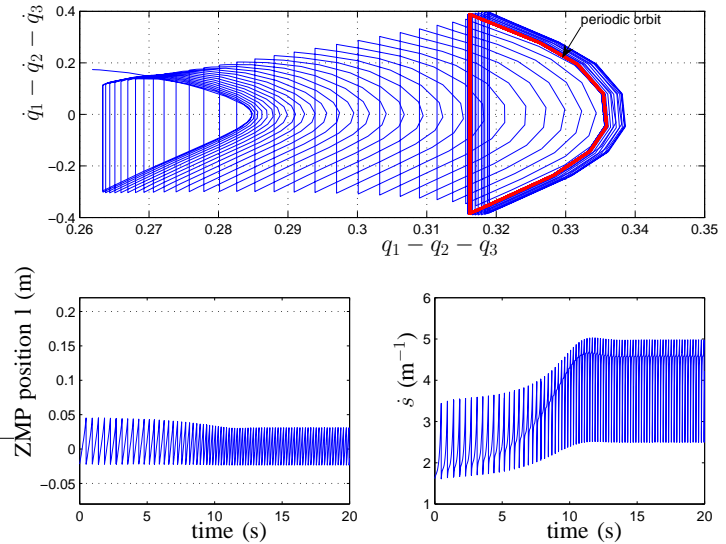

Fig. 6. The convergence toward a periodic motion is observed in simulation with the proposed control law, with modeling error. a) The torso evolution is drawn in its phase plane (the absolute torso velocity with respect to the absolute torso orientation); it tends toward a limit cycle. (b) The horizontal position of the ZMP with respect to $s, l(s)$, tends toward a periodic evolution different from Figure 5(b): the ZMP excursion is smaller than the expected one. (c) $\dot{s}(s)$ tends toward a periodic evolution different from Figure 5(c): the new cycle is faster than the nominal one.

1).

\section{Effect of the ZMP evolution}

\begin{tabular}{|c|c|c|c|c|c|c|}
\hline$l_{\min }$ & $l_{\max }$ & $\zeta^{*}$ & $\frac{Z_{m}}{\delta_{I}^{2}}$ & $\left(\delta(1) \delta_{I}\right)^{2}$ & $\dot{s}_{c}(1)$ & $T[s]$ \\
\hline-0.05 & 0.05 & 943.02 & 149.69 & 0.613 & 3.86 & 0.401 \\
-0.04 & 0.06 & 823.92 & 175.44 & 0.621 & 3.62 & 0.437 \\
-0.03 & 0.07 & 699.48 & 202.78 & 0.630 & 3.36 & 0.488 \\
-0.02 & 0.08 & 569.18 & 231.61 & 0.639 & 3.04 & 0.569 \\
-0.01 & 0.09 & 432.40 & 261.85 & 0.648 & 2.66 & 0.733 \\
0 & 0.10 & 288.45 & 293.42 & \multicolumn{2}{|c|}{ No periodic motion } \\
0.01 & 0.11 & 136.53 & 326.23 & \multicolumn{4}{|c|}{ No periodic motion } \\
\hline
\end{tabular}

TABLE III

THE EFFECT OF THE ZMP EVOLUTION

The evolution of the ZMP throughout the step affects the existence and stability of the periodic motion obtained with the proposed control law. To illustrate this point, we consider various linear evolutions of the position of the ZMP with different average values, $\left(\frac{l^{d}(1)+l^{d}(0)}{2}\right)$, while holding constant the net change in the position of the ZMP, $l^{d}(1)-l^{d}(0)=10$ $\mathrm{cm}$. Table III presents the main properties of the periodic motion and of the control law with respect to the variation of the average value of the ZMP position during one step. Placing the average position of the ZMP closer to the toe leads to larger values of $\left(\delta(1) \delta_{I}\right)^{2}$ and smaller values of $\zeta^{*}$ and average walking speed. When the center of mass is in front of the ZMP, the moment arm due to gravity speeds up the motion. When the center of mass is behind the ZMP, the moment arm due to gravity slows down the motion. When the average position of the ZMP is moved forward, the portion of the step where gravity speeds up the motion decreases, and thus the average walking speed decreases.

In the last two rows of Table III, the value of $\zeta^{*}$ is less than the minimum value necessary to complete a step (i.e., 

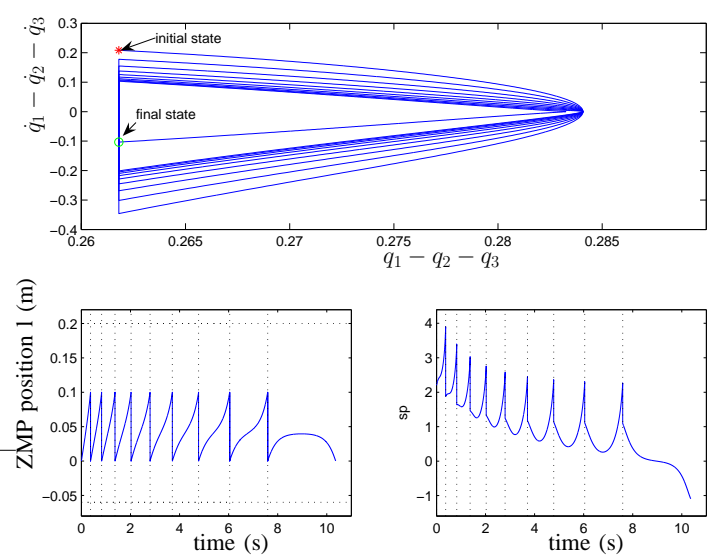

Fig. 7. The motion of the robot is not stable as it does not converge to a periodic motion, but the position of the ZMP remains strictly within the convex hull of the stance foot.

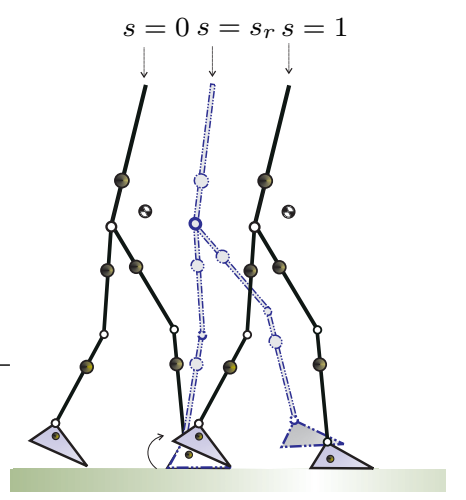

Fig. 8. A walking cycle that includes foot rotation. The single support phase can be decomposed into a flat foot sub-phase and a foot rotation sub-phase. The cycle is completed by an impact phase.

it does not satisfy (27)), and consequently a walking motion cannot be produced. If the control law is used for this case, the behavior shown in Figure 7 is obtained. Perfect tracking of the joint path is observed and the position of the $Z M P$ satisfies at each time instance the condition of non-rotation of the feet (see Figure 7 lower left part), though the motion of the robot is unstable; the robot does not fall down, but it comes to a stop.

\section{WALKING WITH FOOT ROTATION}

The objective in this section is to study how the proposed control law and the associated stability conditions can be extended to the case of a gait that includes a foot rotation subphase [21]. This sub-phase is a normal part of human walking, but humanoid robots generally do not include this sub-phase because it renders the control problem more difficult. The considered gait is described in Figure 8. The double support phase is reduced to an impact phase. This choice has been made since with our model based on rigid bodies, we cannot obtain a non-instantaneous double support phase after the impact if the velocity of the swing leg at impact is nonzero.

The initiation of rotation about the toe is decided by a control action. The reference paths for the joint variables are expressed as functions of $s$ as before. A complete single

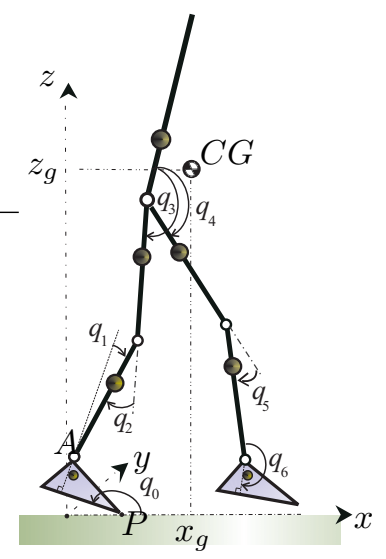

Fig. 9. Generalized coordinates for the foot rotation sub-phase. The rotation about the toe is described by variable $q_{0}$

support phase is achieved for $s=0$ to $s=1$. The transition from the flat-foot sub-phase to the foot-rotation sub-phase occurs for a given value of $s$ denoted $s_{r}$, such that $0<s_{r}<1$.

The Foot Rotation Indicator (FRI) [19] differs from the ZMP by the fact that it can quantify the rotation of the foot. Hence, it could be attractive to control the FRI instead of the ZMP, and to prescribe the foot rotation via the FRI. However, it is difficult to connect the kinematic evolution of the foot to the FRI, which is the reason that we consider the two phases separately.

\section{A. Control during the rotation sub-phase}

From the control point of view, the main difference is that during the flat-foot sub-phase, the evolution of the ZMP can be chosen, whereas during the foot rotation sub-phase, the position of the ZMP is at the contact point between the toe and the ground. The difference is therefore essentially a modeling issue because a supplementary variable, denoted here as $q_{0}$, must be added to describe the configuration of the robot during the rotation sub-phase; see Figure 9. The augmented configuration vector is denoted $q_{r}=\left[q_{0}, q^{T}\right]^{T}$.

During the foot rotation sub-phase, the dynamic model becomes

$$
M_{r}\left(q_{r}\right) \ddot{q}_{r}+h_{r}\left(q_{r}, \dot{q}_{r}\right)=\left[\begin{array}{c}
0 \\
\Gamma
\end{array}\right],
$$

where $M_{r}\left(q_{r}\right)$ is a $(7 \times 7)$ matrix and the vector $h_{r}\left(q_{r}, \dot{q}_{r}\right)$ contains the centrifugal, Coriolis and gravity forces. The first row of (33) corresponds to the passive rotation about the toe, which can also be expressed as

$$
\dot{\sigma}_{P}=m g\left(x_{g}-l_{d}\right)
$$

or by (6) with $l=l_{d}$ (see Figure 1). The first row of the dynamic model (33) also yields the required acceleration $\ddot{s}$ so that $\ddot{q}_{r}$ satisfies the dynamic model. To have the desired closed-loop behavior for the joints, $\ddot{s}$ must be such that

$$
M_{r 1}\left(q_{r}\right)\left(\frac{d q_{r}^{d}(s)}{d s} \ddot{s}+v_{r}\right)+h_{r 1}\left(q_{r}, \dot{q}_{r}\right)=0,
$$

where the index 1 denotes the first row. Thus

$$
\ddot{s}=-\frac{M_{r 1}\left(q_{r}\right) v_{r}+h_{r 1}\left(q_{r}, \dot{q}_{r}\right)}{M_{r 1}\left(q_{r}\right) \frac{d q_{r}^{d}(s)}{d s}} .
$$


The last 6 rows of the dynamic model yield the torques required to track a desired path, as in the second equation of (18).

\section{B. Stability study}

The evolution of the angular momentum $\sigma_{P}$ during one step is studied in order to determine the stability of the gait.

As mentioned in Section IV, during the flat-foot sub-phase, the angular momentum evolution is described by (20) and the variable $\zeta(s)$ evolves as in (25). At the transition between the flat-foot sub-phase and foot-rotation sub-phase, the ZMP position changes, and thus the angular momentum about the ZMP changes. We denote by $\sigma_{P}\left(s_{r}^{-}\right)$, the angular momentum at the end of the flat-foot sub-phase, and by $\sigma_{P}\left(s_{r}^{+}\right)$, the angular momentum at the beginning of the foot-rotation sub-phase. The configuration and velocity of the robot are continuous at this transition.

At the end of the flat foot sub-phase, we have

$$
\zeta\left(s_{r}^{-}\right)=\delta^{2}\left(s_{r}\right) \zeta(0)-\Phi\left(s_{r}\right) .
$$

Using the transfer of angular momentum equation, the change in momentum can be written as

$$
\sigma_{P}\left(s_{r}^{+}\right)=\sigma_{P}\left(s_{r}^{-}\right)+m \dot{z}_{g}\left(s_{r}\right)\left(l_{d}-l^{d}\left(s_{r}\right)\right) .
$$

On the zero dynamics (19), the velocity $\dot{z}_{g}$ can be expressed as a function of $\sigma_{P}\left(s_{r}^{-}\right)$using (21)

$$
\dot{z}_{g}\left(s_{r}\right)=\frac{\frac{\partial z_{g}\left(q^{d}\left(s_{r}\right)\right)}{\partial q} \frac{d q^{d}\left(s_{r}\right)}{d s}}{I\left(s_{r}^{-}\right)} \sigma_{P}\left(s_{r}^{-}\right) .
$$

Therefore,

$$
\sigma_{P}\left(s_{r}^{+}\right)=\delta_{S_{r}} \sigma_{P}\left(s_{r}^{-}\right)
$$

with

$$
\delta_{S_{r}}=1+m \frac{\frac{\partial z_{g}\left(q^{d}\left(s_{r}\right)\right)}{\partial q} \frac{d q^{d}\left(s_{r}\right)}{d s}\left(l_{d}-l^{d}\left(s_{r}\right)\right)}{I\left(s_{r}^{-}\right)} .
$$

For the variable $\zeta$, at the beginning of the foot-rotation subphase we have

$$
\zeta\left(s_{r}^{+}\right)=\delta_{S_{r}}^{2} \delta^{2}\left(s_{r}\right) \zeta(0)-\delta_{S_{r}}^{2} \Phi\left(s_{r}\right) .
$$

During the foot-rotation sub-phase, the ZMP position is $l_{d}$, which has been selected to be constant. Consequently, on the zero dynamics, the angular momentum evolves according to (34). Using the same principle as in Section IV, if there exists a solution beginning with $s=s_{r}, \dot{s}\left(s_{r}\right)>0$ and ending with $s\left(t_{f}\right)=1, \dot{s}\left(t_{f}\right)>0$, we have

$$
\frac{d \sigma_{P}}{d s}=m g\left(x_{g}\left(q^{d}(s)-l_{d}(s)\right) \frac{I(s)}{\sigma_{P}} .\right.
$$

Applying the change of variable, $\zeta(s)=\frac{1}{2} \sigma_{P}^{2}$, this equation becomes

$$
\frac{d \zeta}{d s}=m g I(s)\left(x_{g}\left(q^{d}(s)-l_{d}(s)\right),\right.
$$

and hence, for $s_{r} \leq s \leq 1$,

$$
\zeta(s)=\zeta\left(s_{r}^{+}\right)-\Phi_{t}(s),
$$

where

$$
\Phi_{t}(s)=-m g \int_{s_{r}}^{s} I(\tau)\left(x_{g}\left(q^{d}(\tau)\right)-l_{d}(\tau)\right) d \tau .
$$

This expression can be combined with (42), yielding

$$
\zeta(s)=\delta_{S_{r}}^{2} \delta^{2}\left(s_{r}\right) \zeta(0)-\delta_{S_{r}}^{2} \Phi\left(s_{r}\right)-\Phi_{t}(s) .
$$

Finally, since the expressions for $\zeta$ are similar during the flatfoot and foot-rotation sub-phases, they can be represented by a single expression, for $0 \leq s \leq 1$, namely

$$
\zeta(s)=\delta_{r}^{2}(s) \zeta(0)-\Phi_{r}(s)
$$

with

$$
\begin{aligned}
& \delta_{r}(s)=\left\{\begin{aligned}
\delta(s) & \text { for } \quad 0 \leq s \leq s_{r}^{-} \\
\delta_{S_{r}} \delta\left(s_{r}\right) & \text { for } \quad s_{r}^{+} \leq s \leq 1
\end{aligned}\right. \\
& \Phi_{r}(s)=\left\{\begin{array}{rll}
\Phi(s) & \text { for } \quad 0 \leq s \leq s_{r}^{-} \\
\delta_{S_{r}} \Phi\left(s_{r}\right)+\Phi_{t}(s) & \text { for } \quad s_{r}^{+} \leq s \leq 1 .
\end{array}\right.
\end{aligned}
$$

Remark on Stability Analysis: Because the behavior of $\zeta$ along a single support phase has been expressed in exactly the same form as in the first study concerning fully actuated walking, and because the impact equations are similar due to the previous supporting leg leaving the ground, Theorems 1,2 and 3 and the Corollary can also be restated for this more complex gait, and analogous conditions for existence and stability are obtained by replacing $\delta(s)$ and $\Phi(s)$ by $\delta_{r}(s)$ and $\Phi_{r}(s)$, respectively.

\section{Simulation results}

The control law is evaluated here for the periodic path depicted in the stick diagram of Figure 10. The joint path $q^{d}(s)$ is defined with a degree four polynomial in $s$ for the flat-foot sub-phase, and a degree three polynomial in $s$ for the foot-rotation sub-phase. These joint references have been adjusted by hand, starting from the references presented in Figure 3. Some optimal motions that include the foot-rotation sub-phase can also be defined using the methodology given in [29] but this work is not within the scope of the present paper. In [29], it is also shown that from the energy efficiency point of view, the introduction of a foot-rotation sub-phase is efficient for fast walking.

In the simulation presented here, the transition between the two sub-phases was selected to occur at $s=s_{r}=0.7$, the desired evolution for $l^{d}$ and $q^{d}(s)$ are :

$$
\begin{cases}q^{d}(s)=a_{0}+a_{1} s+a_{2} s^{2}+a_{3} s^{3}+a_{4} s^{4} & 0 \leq s \leq 0.7 \\ l^{d}(s)=-0.03+0.1 s & \\ q_{r}^{d}(s)=b_{0}+b_{1} s+b_{2} s^{2}+b_{3} s^{3} & 0.7<s \leq 1 \\ l^{d}(s)=l_{d}=0.2 & \end{cases}
$$

The vectors $a_{i}$ are determined so as to join an initial configuration $q^{d}(0)$, an intermediate configuration $q^{d}(0.35)$, a final configuration $q^{d}(0.7)$, an initial velocity direction $\frac{d q^{d}(0)}{d s}$ and a final velocity direction $\frac{d q^{d}(0.7)}{d s}$ for the first sub-phase. The vectors $b_{i}$ are calculated to join an initial configuration 
$q_{r}^{d}(0.7)$, a final configuration $q_{r}^{d}(1)$, an initial velocity $\frac{d q_{r}^{d}(0.7)}{d s}$ and a final velocity $\frac{d q_{r}^{d}(1)}{d s}$ for the first sub-phase. In the first sub-phase, no reference is defined for the joint $q_{0}$, thus the notation $q$ and $q_{r}$ are used to define the configuration coordinates. To have continuity in the reference in position and velocity direction $q^{d}(0.7), \frac{d q^{d}(0.7)}{d s}$ and $q_{r}^{d}(0.7), \frac{d q_{r}^{d}(0.7)}{d s}$ describe the same state of the robot. The final configuration of the step, $q_{r}^{d}(1)$, corresponds to the initial one $q^{d}(0)$ with an exchange of the legs. These key values to describe the reference trajectory are given in the Table IV.

\begin{tabular}{|c|c|c|c|c|c|c|}
\hline$q^{d}(0)$ & $\frac{d q^{d}(0)}{d s}$ & $q^{d}(0.35)$ & $q_{r}^{d}(0.7)$ & $\frac{d q_{r}^{d}(0.7)}{d s}$ & $q_{r}^{d}(1)$ & $\frac{d q_{r}^{d}(0.7)}{d s}$ \\
\hline & & & 3.1416 & 0 & 2.9671 & 0 \\
-0.0438 & 2.3798 & 0.1815 & 0.2509 & 0.3562 & 0.5014 & 1.1565 \\
0.3707 & 2.1298 & 0.3546 & 0.1039 & -0.3722 & 0.1957 & 0.6523 \\
2.4653 & 0.0955 & 2.6858 & 3.0086 & 0.7642 & 3.1855 & 0.4003 \\
3.1855 & 0.3248 & 2.9151 & 2.4862 & -0.8358 & 2.4653 & 0.3981 \\
0.1957 & 0.2862 & 0.5306 & 0.7626 & -0.1376 & 0.3707 & 0.3084 \\
2.8148 & -1.7654 & 2.5548 & 2.7543 & 1.0093 & 3.1854 & -1.2468 \\
\hline
\end{tabular}

TABLE IV

THE REFERENCES FOR THE JOINTS ARE BASED ON THESE KEY VECTORS

The rotation occurs due to a control action corresponding to a discontinuity on the torque. The torques are such that an acceleration of $q_{0}$ appears and that the ZMP is at the contact point between the toe and the ground.

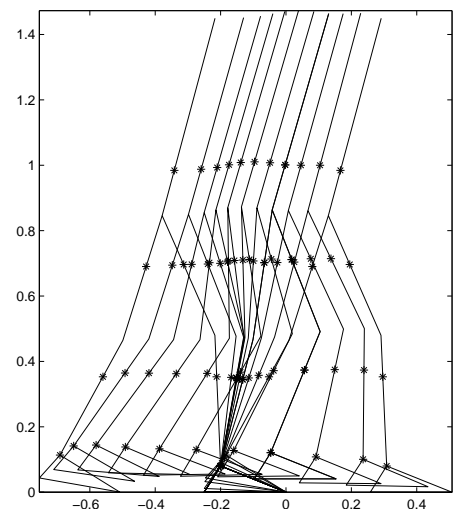

Fig. 10. The stick diagram of the desired trajectory. The configuration of the robot is drawn for $s=0,0.1,0.2 \ldots, 0.9,1$. For $0 \leq s \leq 0.7^{-}$, the supporting foot is flat on the ground. For $0.7^{+} \leq s \leq 1$, the supporting foot rotates.

The evolution of $\delta_{r}(s)$ and $\Phi_{r}(s)$ are depicted in Figure 11. Their final values, $\Phi_{r}(1)=-124$ and $\delta_{r}(1)^{2}=0.9585$, are useful for constructing the Poincare map. The behavior of $\zeta$ during the impact is defined by $\delta_{I}^{2}$, which is equal to 0.6440. The minimum value of $\zeta$ for which a step can be achieved is $\frac{Z_{m}}{\delta_{I}}=198$. The periodic motion is given by (30). The fixed point is $\zeta^{*}=324$. The slope of the Poincare return map $\rho$ is $\left(\delta(1) \delta_{I}\right)^{2}=0.6161$, and because it is less than 1 , the corresponding periodic walking motion is exponentially stable. The stability arises from the effect of the impact because $\delta_{r}(1)^{2}$ is close to 1 .

The forward walking speed of the robot will be slower when the gait includes rotation about the toe than when this subphase does not exist. During the rotation sub-phase, the ZMP
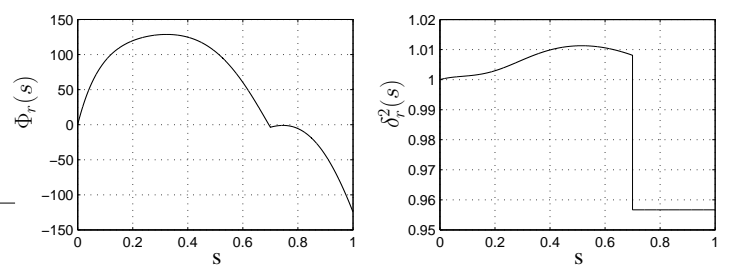

Fig. 11. The evolution of $\zeta$ during the single support phase is characterized by the functions $\Phi_{r}(s)$ and $\delta_{r}^{2}(s)$. During the flat-foot sub-phase, an evolution close to that of Figure 4 is observed. At the transition between the sub-phases, a jump occurs due to the term $\delta_{s_{r}}^{2}$; however, for this illustration, $\delta_{s_{r}}^{2}$ is close to 1 and thus this jump is difficult to see for $\phi_{r}(s)$. Since at the transition the position of the ZMP changes from $0.07 \mathrm{~m}$ to $0.2 \mathrm{~m}$, the center of mass that was in front of the ZMP becomes behind the ZMP, and thus $\Phi_{r}$ increases slightly just after the transition. $\delta_{r}(s)$ is constant during the foot rotation sub-phase.

is at the forward edge of the foot, the decrease of $\Phi_{r}$ is less than if the ZMP was inside the sole of the feet (see Figure 11 ), and the resulting value of $\zeta^{*}$ corresponding to the periodic motion decreases.
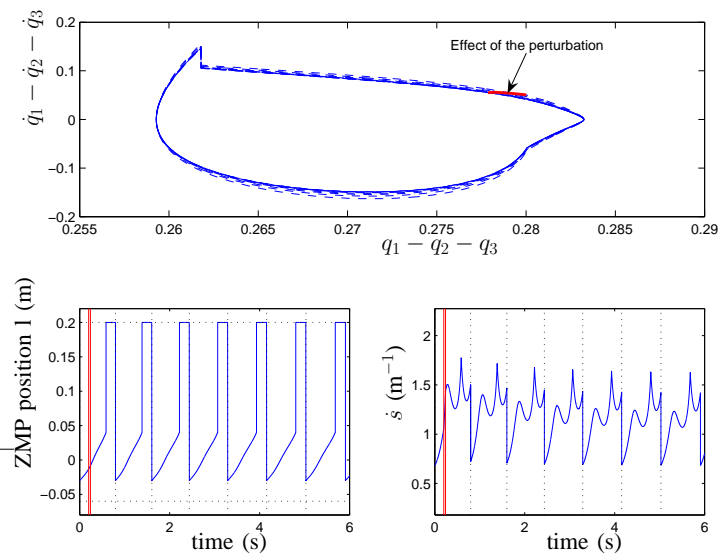

Fig. 12. The convergence towards a periodic motion is observed in simulation with the proposed control law.

A simulation was done for ten steps, assuming no modeling error and initializing the state of the robot on the periodic orbit. A horizontal force of $100 \mathrm{~N}$ is applied at the center of mass for $0.2 s<t<0.24 s$; see Figure 12. Convergence toward a periodic motion was obtained for each of the six joints of the robot. As an illustration, the evolution of the angle of the torso is depicted in Figure 12-a. The same convergence is also evident in the evolution of the position of the ZMP with respect to time in Figure 12-b; for each step, its evolution is linear from $-0.03 m$ to $0.04 m$ during the flat-foot sub-phase and then there is a discontinuity to achieve $l^{d}=0.2 \mathrm{~m}$. Figure 12-c presents the evolution of $\dot{s}$ with respect to time; it clearly converges toward a periodic motion.

\section{Interesting next steps}

The simulation results have shown the effectiveness of the control law. The evaluation of this approach on a prototype would be of great interest. This has not yet been attempted because a planar biped with feet is not available for such experiments. The proposed control law required a computed 
torque control that may be difficult to implement on a real robot. A PD controller can be derived from this control approach instead of the computed torque control. The most important point is to define the reference motion not as a predefined function of time, but as a function of desired kinematic evolution of the position of the ZMP. If the integration of the variable $\ddot{s}$, needed to define the reference path, is difficult in practice, a physical monotonic variable based on the measurable state of the robot, for example the angle of a virtual stance leg, can be used instead of $s$. Additional information about these practical implementation issues are are given in [32].

The extension to a 3D biped would also be interesting. Two directions could be chosen to do this extension.

Suppose the robot is fully actuated. The main interest of the proposed strategy is to show that a temporal modification of the joint reference motion allows us to correct the position of the ZMP. In the case of a 3D motion, the position of the ZMP has to be controlled in two directions (frontal and sagittal), and the temporal modification gives only one degree of freedom. It is not possible with this degree of freedom to track a prescribed motion of the ZMP in these two directions, but it could be possible to maintain the ZMP inside a prescribed sub-surface of the complete sole. In the case of planar motion, how to keep the ZMP in a prescribed area is described in [9]. The introduction of rotation phase about the toe for $3 \mathrm{D}$ motion is similar to the 2D case because only one free degree of rotation appears.

The control strategy of this paper has been built upon a control approach developed for point-contact planar robots [5]. A control strategy for a point-contact 3D robot is now under development [23].

\section{CONCLUSION}

For a planar biped, a control strategy was proposed based on tracking a reference path in the joint space instead of a reference function of time. This allows the simultaneous control of the path positions of the joints and the ZMP. The biped adapts its time evolution according to the effect of gravity. A stability study of the robot's time evolution has been given for a fully actuated gait and a gait that includes a foot rotation sub-phase (i.e., an underactuated sub-phase). Walking with more human-like characteristics can be handled by our control law. Easily testable analytical conditions have been presented for the existence and uniqueness of a periodic motion and for the orbital exponential stability of a periodic motion. Since the stability conditions are based on inequalities, a natural robustness with respect to modeling errors and external perturbation exists.

\section{REFERENCES}

[1] A.P. Aguiar, J.P. Hespanha, and P.V. Kokotovic. Path-following for nonminimum phase systems removes performance limitations. IEEE Transactions on Automatic Control, 50(2):234 - 239, 2005.

[2] S. P. Bhat and D. S. Bernstein. Continuous finite-time stabilization of the translational and rotational double integrators. IEEE Transaction on Automatic Control, 43(5):678-682, 1998.

[3] Fu. Chenglong, M. Shuai, and K. Chen. Proving asymptotic stability of dynamic walking for a five-link biped robot with feet. In IEEE Conference on Robotics, Automation and Mechatronics, december 2006.
[4] C. Chevallereau, G. Abba, Y. Aoustin, F. Plestan, E. R. Westervelt, C. Canudas-de Witt, and J. W. Grizzle. Rabbit: A testbed for advanced control theory. IEEEControl System Magazine, 23(5):57-78, 2003.

[5] C. Chevallereau, Formal'sky A. M., and D. Djoudi. Tracking of a joint path for the walking of an under actuated biped. Robotica, 22(1):15-28, 2004.

[6] J. H. Choi. Model-based Control and Analysis of Anthropomorphic Walking. PhD thesis, University of Michigan, 2005.

[7] Jun Ho Choi and J.W. Grizzle. Planar bipedal walking with foot rotation. In Proceedings of the American Control Conference, pages 4909 - 4916, June 2005.

[8] O. Dahl and L. Nielsen. Torque-limited path following by online trajectory time scaling. IEEE Trans. on Automat. Contr, 6(5):554-561, 1990.

[9] D. Djoudi and C. Chevallereau. Fast motions in Biomechanics and Robotics, chapter Stability analysis of bipedal walking with control or monitoring of the center of pressure, pages 95-120. LNCIS. Springer, Heidelberg, 2006.

[10] J. W. Grizzle, G. Abba, and F. Plestan. Asymptotically stable walking for biped robots: analysis via systems with impulse effects. IEEE Trans. on Automat. Contr., 46:51-64, 2001.

[11] K. Hirai, M. Hirose, Y. Haikawa, and T. Takenaka. The development of honda humanod robot. In Proc. of the IEEE International Conference on Robotics and Automation, pages 1321-1326, Leuven, Belgium, 1998.

[12] Y. Hürmüzlü. Dynamics of bipedal gait-Part 1: objective functions and the contact event of a planar five-link biped. Journal of Applied Mechanics, 60(2):331-6, 1993.

[13] Y. Hürmüzlü. Dynamics of bipedal gait-Part 2: stability analysis of a planar five-link biped. Journal of Applied Mechanics, 60(2):337-43, 1993.

[14] S. Kagami, F. Kanehiro, Y. Tamiya, M. Inaba, and H. Inoue. Autobalancer: An online dynamic balance compensation scheme for humanoid robots. In Proceedings of the 4th International Workshop on Algorithmic Foundation on Robotics, 2000.

[15] M. Morisawa, Y. Fujimoto, T. Murakami, and K. Ohnishi. A walking pattern generation for biped robot with parallel mechanism by considering contact force. In Proc. of the IEEE Annual Conference on Industrial Electronics Society, Denver, CO, pages 2184-9, 2001.

[16] J. H. Park. Impedance control for biped robot locomotion. IEEE Transactions on Robotics and Automation, 17(6):870-82, December 2001

[17] J. H. Park and H. C. Cho. An on-line trajectory modifier for the base link of biped robots to enhance locomotion stability. In Proceedings of IEEE Int. Conf. on Robotics and Automation, pages 3353-3358, 2000.

[18] F. Pfeiffer, K. Loffler, and M. Gienger. The concept of jogging johnie. In IEEE Int. Conf. on Robotics and Automation, 2002.

[19] M. B. Popovic, A. Goswami, and H. Herr. Ground reference points in legged locomotion: Definitions, biological trajectories and control implications. I. J. Robotic Res., 24(12):1013-1032, 2005.

[20] Huang Q., Y. Nakamura, and T. Inamura. Humanoids walk with feedforward dynamic pattern and feedback sensory reflection. In Proceedings of IEEE Int. Conf. on Robotics and Automation, pages 4220-4225, 2001.

[21] P. Sardain and G. Bessonnet. Gait analysis of a human walker wearing robot feet as shoes. In Proc. of the 2001 IEEE International Conference on Robotics and Automation, Seoul, Korea, pages 2285-92, may 2001.

[22] C.-L. Shih. Ascending and descending stairs for a biped robot. IEEE Transactions on Systems, Man and Cybernetics, 29(3):255-68, 1999.

[23] C-L. Shih, J. W. Grizzle, and Chevallereau C. Asymptotically stable walking of a simple underactuated $3 \mathrm{~d}$ bipedal robot. In Reprint 33rd Annual Conference of IEEE Industrial Electronics (IECON 2007), pages 1404-1409, Taipei, Taiwan, November 2007.

[24] M. W. Spong and F. Bullo. Controlled symmetries and passive walking. IEEE Transactions on Automatic Control, 50(7):1025-31, 2005.

[25] T. Sugihara and Y. Nakamura. Whole-body cooperative balancing of humanoid robot using cog jacobiano. In Proceedings of the 2002 IEEE/RSJ International Conference on Intelligent Robots and Systems, pages 2575-2580, Lausanne, Switzerland, Oct, 2002.

[26] T. Sugihara, Y. Nakamura, and Inoue H. Realtime humanoid motion generation through $\mathrm{zmp}$ manipulation based on inverted pendulum control. In Proceedings of IEEE Int. Conf. on Robotics and Automation, pages 1404-1409, 2002.

[27] T. Takahashi and A. Kawamura. Posture control using foot toe and sole for biped walking robot "Ken". In Proc. of the 2002 IEEE International Workshop on Advanced Motion Control, Maribor, Slovenia, pages 43742, 2002. 
[28] A. Takanishi, Y. Egusa, M. Tochizawa, T. Takeya, and I. Kato. Realisation of dynamic walking stabilized with trunk motion. In Proceedings of ROMANSY 7, pages 68-79, 1988.

[29] D. Tlalolini, C. Chevallereau, D. Djoudi, and Y. Aoustin. Optimal reference motions with foot rotation for a biped. In submitted to IEEE Int. Conf. on Robotics and Automation, 2008.

[30] M. Vukobratovic and B. Borovac. Zero moment point -thirty five years of its live. Int. Journal of Humanoid Robotics, 1(1):157-173, 2004.

[31] M. Vukobratovic and J. Stepanenko. "on the stability of anthropomorphic systems". Mathematical Biosciences, 15(1):1-37, 1972.

[32] E.R. Westervelt, J.W. Grizzle, C. Chevallereau, J.H. Choi, and B. Morris. Feedback Control of Dynamic Bipedal Robot Locomotion. Taylor and Francis/CRC Press, 2007.

[33] E.R. Westervelt, J.W. Grizzle, and D.E. Koditschek. Hybrid zero dynamics of planar biped walkers. IEEE Transaction on Automatic Control, 48(1):42-56, 2003.

[34] K. Y. Yi. Walking of a biped robot with compliant ankle joints: implementation with KUBCA. In Proc. of the 2000 IEEE International Conference on Decision and Control, Sydney, Australia, volume 5, pages 4809-14, 2000.

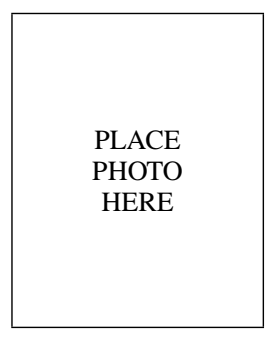

Jessy W. Grizzle is the Jerry W. and Carol L. Levin Professor of Engineering at the University of Michigan. He received the Ph.D. in electrical engineering from The University of Texas at Austin in 1983 and in 1984 held an NSF-NATO Postdoctoral Fellowship in Science in Paris, France at the CNRS . Since September 1987, he has been with The University of Michigan, Ann Arbor, where he is a Professor of Electrical Engineering and Computer Science. His research interests have often focused on theoretical aspects of nonlinear systems and control, including geometric methods for continuous- and discrete-time systems, and observer design in discrete-time. He has been a consultant in the automotive industry since 1986, where he jointly holds sixteen patents dealing with emissions reduction through improved controller design. His current interest in bipedal locomotion grew out of a sabbatical in Strasbourg, France. Prof. Grizzle was elected Fellow of the IEEE in 1997. His awards include: with K.L. Dobbins and J.A. Cook (Ford Motor Company), 1992 Paper of the Year Award from the IEEE Vehicular Technology Society; with G. Abba (Univ. of Metz, France) and F. Plestan (Ecole Centrale, Nantes, France), the 2002 Axelby Award from the IEEE Control Systems Society; and with J. Sun (Univ. of Michigan) and J. Cook (Ford), the 2003 IEEE Control Systems Society Technology Award. $\mathrm{He}$ has served as Associate Editor for the Transactions on Automatic Control and Systems \& Control Letters, Publications Chairman for the 1989 CDC, on the Control Systems Society's Board of Governors in 1997-1999, Associate Editor for Automatica 2002-2005, AACC Award Committee (2003-2005), and is currently an Associate Editor at Large for the IEEE Transactions on Automatic Control.

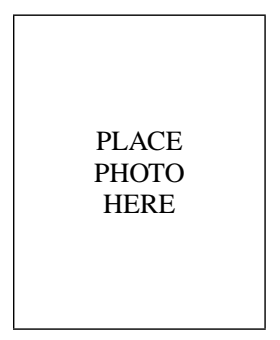

Christine Chevallereau graduated from Ecole Nationale Supérieure de Mécanique, Nantes in 1985, and received the Ph.D. degree in Control and Robotics from Ecole Nationale Supérieure de Mécanique, Nantes, France in 1988. Since 1989, she has been with the CNRS in the Institut de Recherche en Communications et Cybernetique de Nantes. Her research interests include modeling and control of robots, especially control of robot manipulators and legged robots.
Dalila Djoudi was born in Algeria in 1978. She obtained the diploma of Electronic Engineering from the Centre Universitaire de Djelfa, Algeria in 2001 and $\mathrm{a} \mathrm{Ph}$. D in Robotics from the Universit de Nantes, France in 2007. Since November 2007, she is an Assistant Professor at the Centre Universitaire de Djelfa, Algeria. Her research interests include control of walking robots and optimal motion for bipeds. 